\title{
Investigate on Chloroform Vapor Induced Growth of Single Crystal of Poly (butylene adipate)
}

\author{
Yunyang Song ${ }^{1, ~ a}$, Haimu Ye ${ }^{1, b}$ and Guiwu $\mathrm{Lu}^{1, \mathrm{c}}$ \\ ${ }^{1}$ Department of Materials Science and Engineering, China University of Petroleum, Beijing 102249, \\ China \\ asongyy1021@126.com, bcuppolymer@163.com, 'lugw@cup.edu.cn
}

Keywords: Poly (butylene adipate), vapor induce crystallization, single crystal.

\begin{abstract}
The phase transition behavior of PBA films under $\mathrm{CCl}_{3}$ vapor at different temperatures was investigated by atomic force microscope (AFM). The height and phase images indicate $\mathrm{CCl}_{3}$ vapor will induce single crystals grow on the surface of PBA film. The vapor induced newly formed PBA single crystal could influence the surface appearance of the background PBA film, make the PBA film uneven (annealing at $25^{\circ} \mathrm{C}$ ). In turn, the crystal phenomenon of the PBA film could also influence the orientation of newly formed PBA single crystal. Strip PBA single crystals would oriented perpendicular to the direction of the radius of the substrate spherulites (annealing at $35^{\circ} \mathrm{C}$ ). Regular shape single crystals would formed when annealed at proper temperature $\left(30^{\circ} \mathrm{C}\right)$.
\end{abstract}

\section{Introduction}

The crystallization of polymer can be achieved by three ways. Among them, the most widely studied way is thermal induced crystallization (form melt or glass state), which is very important in the industry. Another way is stress induce crystallization, the third way is solvent induce crystallization (SINC). SINC is crystalline polymer crystallization under the influence of solvent and vapor. The process includes the following several stages [1]: first, the solvent molecule diffuse into the polymer; second, the solvent molecule interaction with the polymer, which will destory the interaction force between polymer molecules and improve the mobility of polymer chain segment. If the interaction force between solvent and polymer molecules is strong enough, polymer molecules will rearrange itself into crystalline state which is a lower energy state.

As one of the bioplastics, poly(butylene adipate) (PBA) has attracted much attention. (PBA) is a biodegradable polyester with good tensile property. Due to the good crystallinity and polymorphic behavior, PBA is offen chosen as research subject [2,3]. In this study, the crystallization behaviour of PBA under $\mathrm{CCl}_{3}$ vapor is investaged by Atomic Force Microscope (AFM). A growth pattern is proposed based on the results.

\section{Experiment}

PBA was synthesized from 1,4-butanediol and adipic acid by a two-step reaction of esterification and polycondensation in the molten state, and details of the synthesis process were reported previously[4]. The number average molecular weight (Mn) and polydisperse index (PDI), determined by gel permeation chromatography (GPC, Viscotek, M302 TDA), are $5.7 \times 104 \mathrm{~g} / \mathrm{mol}$ and 1.82, respectively. Chloroform $\left(\mathrm{CCl}_{3}\right)$ was purchased from Aladdin Company.

Thin films of PBA about $100 \mathrm{~nm}$ were prepared via spin-coating $1 \mathrm{wt} \%$ chloroform solution onto a freshly cleaved mica surface, then dried at a vacuum oven at $50{ }^{\circ} \mathrm{C}$ for 2 days. The mica is placed on a glass stage which is setted in the middle of a sealed jar. The jar is filled with certain amount of $\mathrm{CCl}_{3}$ and the jar is placed in a water bath to control the temperature of the $\mathrm{CCl}_{3}$.

The AFM figures are recorded under tapping-mode AFM using a Nanoscope III MultiMode AFM (Digital Instruments). Both height and phase images were recorded simultaneously. Typical values for the set-point amplitude ratio were 0.7-0.9. The amplitude of the freely oscillating cantilever was approximately $40 \mathrm{~nm}$. RTESP tips with a resonance frequency of approximately 300 $\mathrm{kHz}$ and a spring constant of about $30 \mathrm{~N} / \mathrm{m}$ were used. 


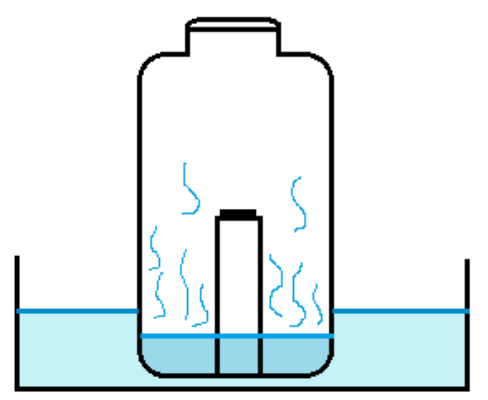

Fig. 1 Scheme of the experiment device

\section{Result and discussion}

Fig. 2 is the AFM height image of the PBA films spin-coated from $\mathrm{CCl}_{3}$ followed by drying at a vacuum oven at $50{ }^{\circ} \mathrm{C}$ for 2 days. It can be seen that the PBA crystallized into spherulite crystal, and the size of the spherulites is about ten micrometers to dozens micrometers. The stripes spreading from the spherulite core indicates the lamellae of the spherulite is edge-on. Since the PBA film is annealed at $50^{\circ} \mathrm{C}$ for 2 days, the crystal form should be $\alpha$ form [5]. The PBA film is used for the vapour induced crystallization experiment. After the film is placed in the sealed jar contained $\mathrm{CCl}_{3}$ solvent, the film is exposed under the $\mathrm{CCl}_{3}$ vapour. The $\mathrm{CCl}_{3}$ vapour pressure is controled by the water bath temperature, higher temperature will prompt more $\mathrm{CCl}_{3}$ heated to evaporation became gas. Different temperature and different time are chosen to observe how the $\mathrm{CCl}_{3}$ vapour will influence the surface structure of PBA crystal, and some typical images in shown below.

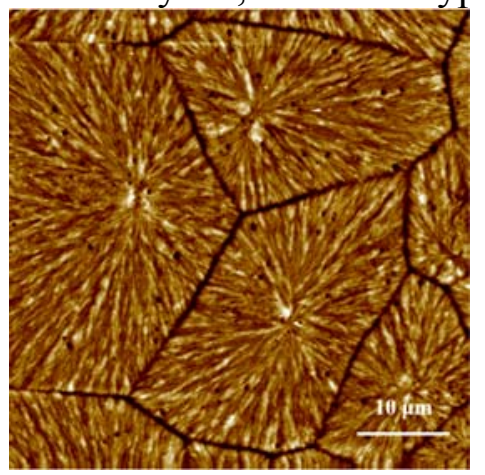

Fig. 2 AFM height image of PBA films spin-coated from $\mathrm{CCl}_{3}$

Fig. $3 \mathrm{a}$ is the AFM height image of the PBA films annealing under $\mathrm{CCl}_{3}$ vapor at $25^{\circ} \mathrm{C}$ for $8 \mathrm{hrs}$. It can be seen that a quite large single crystal is formed on the surface. The single crystal is ribbon like, and gradually gets narrower from middle to tail. It is interesting to note that the single crystal is not flat, but many ups and downs on the surface which is more obvious in the three dimensional height image (Fig. 3b). The scale mark shows that the highest point on the single crystal is $270 \mathrm{~nm}$ higher than the lowest point, it's quite exaggerated since the single crystal is only $20 \mathrm{~nm}$ thick. This illustrates the growth of the single crystal under the $\mathrm{CCl}_{3}$ vapor has a significant effect on the PBA film. The $\mathrm{CCl}_{3}$ vapor dig a hole on the PBA film, and transport material in the hole to its sides forming a raised platform. In order to understand this phenomenon, an explanation is proposed. As shown in Fig. 4, the original PBA film is composed of edge-on chain folded lamella, and a polymer chain may interweave in two or more adjacent lamella which is known as "switchboard" model. The $\mathrm{CCl}_{3}$ vapor will destroy the interaction between the polymer chains, some polymer chains ends on the surface of a lamellar will be separate itself from the lamellar, and recrystallized into a new flat-on lamellar. The crystallization process will drive the polymer chain dissociate form the edge-on lamellar and recrystallization into a new flat-on lamellar, which will induce the edge-on lamellar is rapidly consumed, and the newly formed flat-on lamellar will cover and protect the adjacent lamellar from being dissolved. In general, the arrangement of the molecules in the edge-on lamellar and crystal is the cause of the peculiar surface profile. 

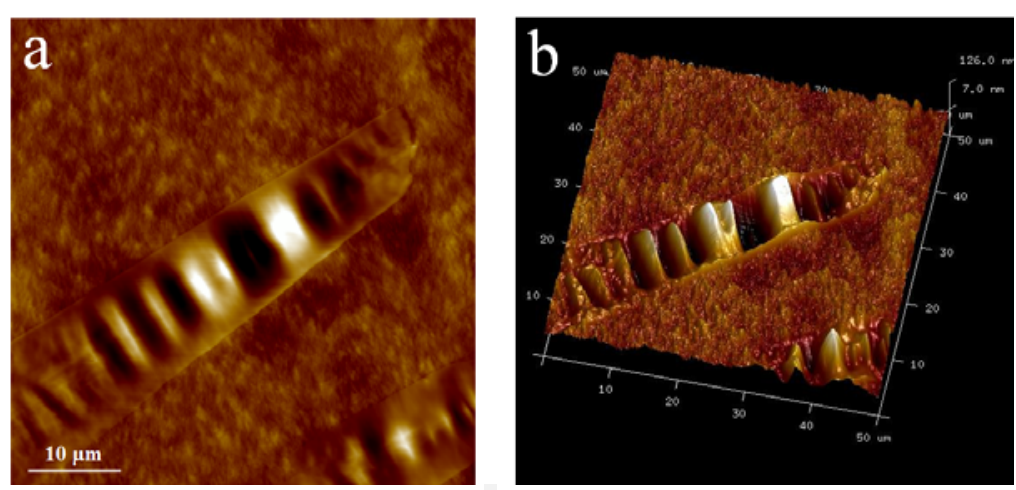

Fig. 3 AFM micrographs (a) two-dimensional (b) three-dimensional height image of the PBA films spin-coated from $\mathrm{CCl}_{3}$ followed by annealing under $\mathrm{CCl}_{3}$ vapor at $25^{\circ} \mathrm{C}$ for $8 \mathrm{hrs}$
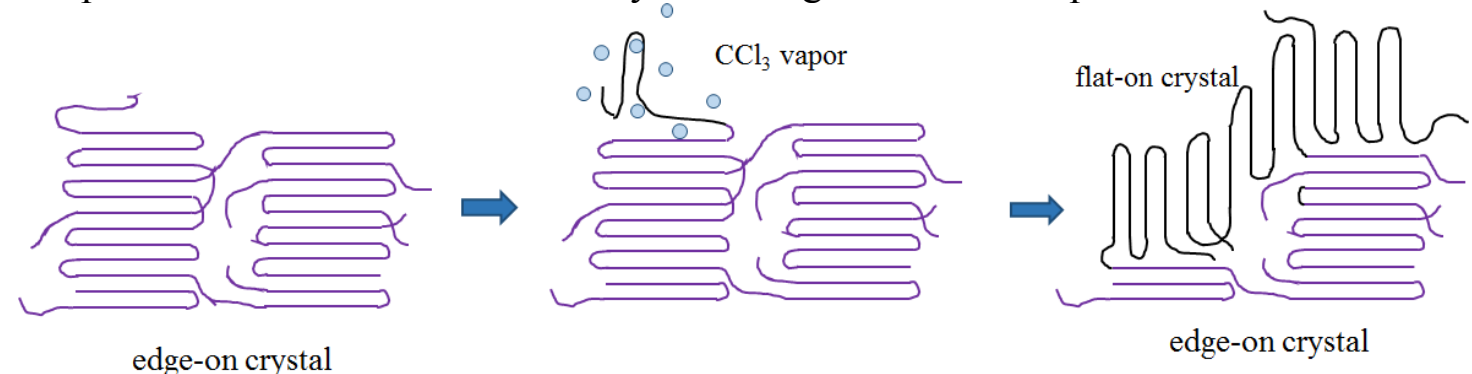

Fig. 4 Scheme of the PBA film recrystallization into single crystal under $\mathrm{CCl}_{3}$ vapor at $25^{\circ} \mathrm{C}$.

Putting the PBA films under $\mathrm{CCl}_{3}$ vapor at $30^{\circ} \mathrm{C}$ for $4 \mathrm{hrs}$, the surface appearance as shown in Fig.5 is quite different from the Fig.3. Fig. 5a is the height image, even different from the original one (fig. 2) it's quite difficult to tell the newly formed single crystal. Some scattered mosaic patterns may suggesting the appearance of single crystal. The phase image (Fig. 5b) is clearly show that many 1-2 $\mu \mathrm{m}$ sized single crystals are grown on the surface. The single crystal is only 4-6 nm thick, which is too thin to to make a distinction with the background PBA film, so the single crystals is hard to distinguished on the height image. The background PBA crystal is edge-on crystal and the newly formed single crystals are flat-on crystal, so that they exposed the lateral surface and chain-folded surface to the AFM probe separately. The big difference of the modulus of the two kinds of lamellar surface result the edge-on crystal and the the flat-on crystal are showing different phase signal. Therefore, the single crystals are outstanding in the phase image. Fig. $5 \mathrm{c}$ is the enlarge picture of Fig. 5b, it can be seen that the newly formed single crystals have straight-line and regular shape. By measuring the angle of the crystal, the angles are close to $60^{\circ}$ or $120^{\circ}$. Previous research shows that the angle between the 110 planes for the $\beta$-form single crystal has such angle degree[6]. It is suggested that the vapor induced PBA crystallization has a preference for $\beta$-form single crystal. 

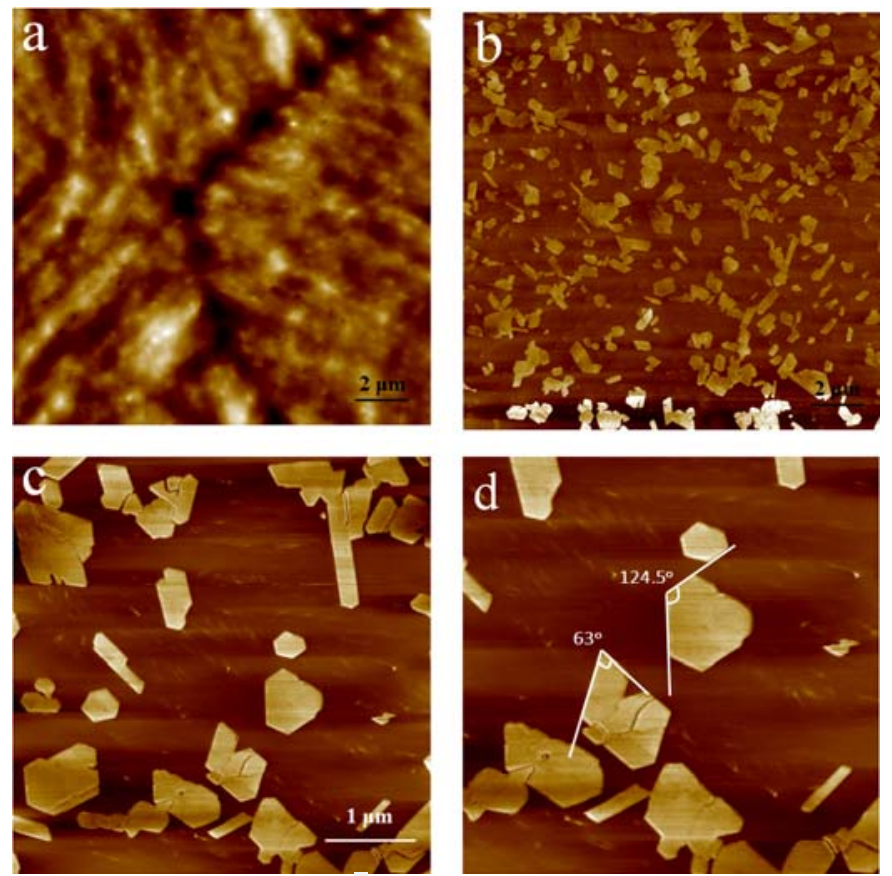

Fig. 5 AFM micrographs (a) height and (b) phase image of the PBA films spin-coated from $\mathrm{CCl}_{3}$ followed by annealing under $\mathrm{CCl}_{3}$ vapor at $30^{\circ} \mathrm{C}$ for $4 \mathrm{hrs}$. (c) the enlarge image of (b), (d) the enlarge image of (c), and the angles of the single crystal are indicated.

The vapor induced newly formed PBA single crystal could influence the surface appearance of the background PBA film, in turn, the crystal phenomenon of the PBA film could also influence the shape of newly formed PBA single crystal. Fig. 6a is the AFM height image of PBA film annealing under $\mathrm{CCl}_{3}$ vapor at $35{ }^{\circ} \mathrm{C}$ for $4 \mathrm{hrs}$. Due to the single crystals are so thin, the shape of the single crystals is not easy to distinguish. It can be seen from the height image that a large size spherulite crystal is formed on the film. Fig. $6 \mathrm{~b}$ is the phase image, a lot of strip single crystal is even distributed. More peculiar is the long axis of those single crystal are almost perpendicular to the direction of the radius of the spherulite crystal. Since the PBA film is composed by edge-on lamellar, and the lamellar is oriented along the direction of the radius. It is suggested that the orientation of the single crystals is related to the growth process of the single crystal. As shown in Fig. 4, the flat-on lamellar is stretch across two or more edge-on lamellar such the strip flat-on lamellar will more likely be perpendicular with the underlying edge-on lamellar.
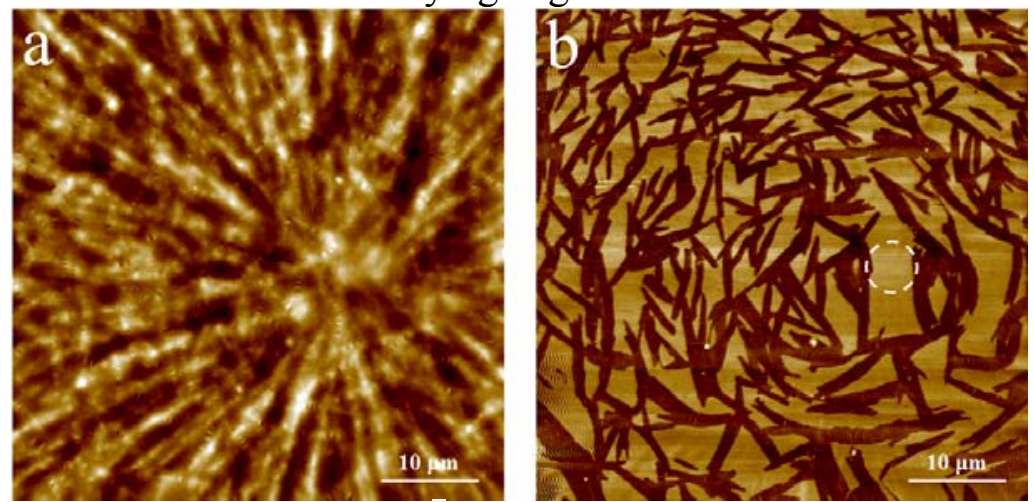

Fig. 6. AFM micrographs (a) height and (b) phase image of the PBA films spin-coated from $\mathrm{CCl}_{3}$ followed by annealing under $\mathrm{CCl}_{3}$ vapor at $35{ }^{\circ} \mathrm{C}$ for $4 \mathrm{hrs}$. The white circle in (b) is used to indicate the center of the spherocrystal.

\section{Summary}

The phase transition behavior of PBA films spin-coated from $\mathrm{CCl}_{3}$ annealing under $\mathrm{CCl}_{3}$ vapor at different temperatures was investigated by atomic force microscope (AFM). The height and phase images indicate $\mathrm{CCl}_{3}$ vapor will induce single crystals grow on the surface of PBA film. By 
annealing the PBA film at $25{ }^{\circ} \mathrm{C}$, the vapor would induce the formation of bigger sized lance-shaped PBA single crystals. And the single crystals could influence the surface appearance of the background PBA film, make the PBA film uneven. Regular shape single crystals would formed when annealed at $30{ }^{\circ} \mathrm{C}$. By annealing the PBA film at $35{ }^{\circ} \mathrm{C}$, strip PBA single crystals would formed and oriented perpendicular to the direction of the radius of the substrate spherulites.

\section{Reference}

[1]. Desai AB, Wilkes GL. Solvent-induced crystallization of polyethylene terephthalate. J PolymSci: PolymSymp. Vol. 46 (1974) No.11, p. 291-319.

[2]. Sun X, Pi F, Zhang J, et al. Study on the Phase Transition Behavior of Poly (butylene adipate) in its Blends with Poly (vinyl phenol). The Journal of Physical Chemistry B. Vol. 115 (2011) No. 9, p. 1950-1957.

[3]. Yang J, Pan P, Hua L, et al. Polymorphic crystallization and phase transition of poly (butylene adipate) in its miscible crystalline/crystalline blend with poly (vinylidene fluoride). Macromolecules. Vol. 43 (2010) No. 20, p. 8610-8618.

[4]. Ye HM, Wang RD, Liu J, et al. Isomorphism in Poly (butylene succinate-co-butylene fumarate) and Its Application as Polymeric Nucleating Agent for Poly (butylene succinate). Macromolecules, Vol. 45(2012) No. 14, p. 5667-5675.

[5]. Gan Z, Kuwabara K, Abe H, et al. Metastability and transformation of polymorphic crystals in biodegradable poly (butylene adipate). Biomacromolecules. Vol. 5 (2004) No. 2, p. 371-378.

[6]. Minke R, Blackwell J. Single crystals of poly (tetramethylene adipate)[J]. Journal of Macromolecular Science, Part B: Physics. Vol. 18 (1980) No. 2, p. 233-255. 